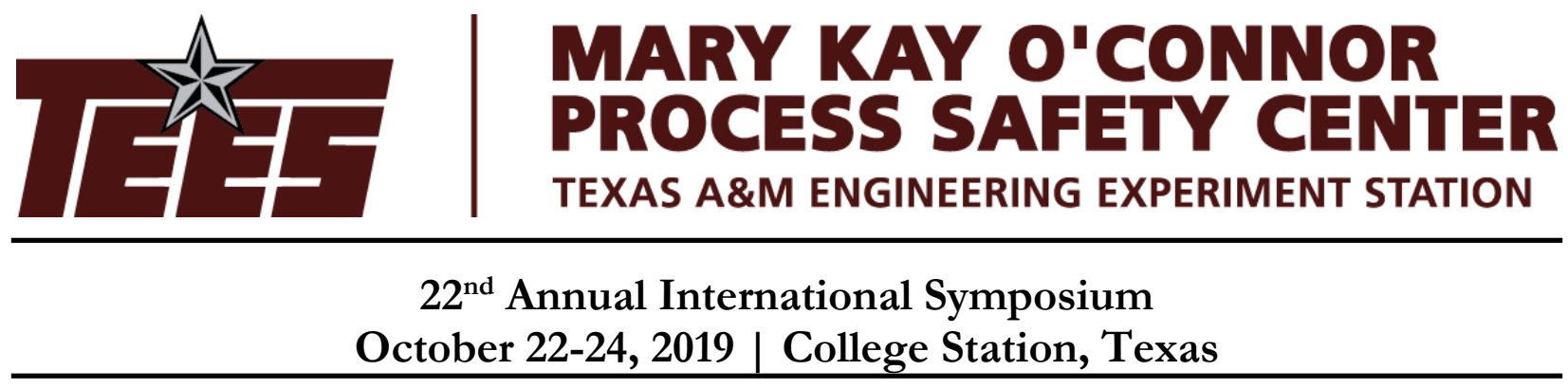

\title{
A New Look at Release Event Frequencies
}

\author{
Benjamin R. Ishii, Jeffrey D. Marx* \\ Quest Consultants Inc. \\ Norman, Oklahoma \\ *Presenter E-mail: jdm@questconsult.com
}

\begin{abstract}
Within the context of a quantitative risk analysis (QRA), the two main constituents used to describe petrochemical risks are, and have always been, consequence and probability. The consequences of hazardous material accidents are easy to apprehend - if a hazard is realized it can injure people or cause fatalities, damage equipment or other assets, or cause environmental damage. Frequencies for these consequences, on the other hand, are not as easy to understand. Process safety professionals develop event frequencies by evaluating historical data and calculating incident rates, which represent, in the QRA context, how often a release of a hazardous material has occurred. Incident rates are further modified by probabilities for various hole sizes, release orientations, weather conditions, ignition timing, and other factors, to arrive at unique event probabilities that are applied in the QRA. This paper describes the development of incident rates from historical database information for various equipment types, as well as defining a methodology for assigning hole size probabilities from the same data, such that a hole size distribution can be assigned within each QRA study. The combination of total incident rates and a hole size distribution relationship can then serve as a foundation within the frequency side of many QRA studies.
\end{abstract}

Keywords: Probability, Frequency, Quantitative Risk Analysis, QRA

\section{Historical Context}

The use of quantitative risk analysis (QRA) within the realm of petrochemical process safety has its roots in the risk assessments of the 1950s and 1960s nuclear industry, as well as the probabilistic risk analysis of the 1960's aerospace programs. It was not until the 1980s, however, that computing power made a fully numeric and sufficiently detailed QRA possible. In the decades since then, QRA has progressed to be more sophisticated, more comprehensive ${ }^{[1]}$, and more adept at providing sensitivity and validation studies with an analysis. 
During the early days of QRA, the consequence analysis portion was viewed as the weak link the models were simplistic and overly conservative, but necessarily so due to the computational resources that were available. As computing power increased over the years, and the consequence models were improved with new techniques and field-scale validations, the frequency assessment became the weaker of the two. A general realization was made by risk analysts that many of the incident rates in published data were nothing more than engineering estimates (guesstimates) and often came with uncertainties that were measured in orders of magnitude (for example, see the "Purple Book" from $\mathrm{TNO}^{[2]}$ or the data presented by the International Association of Oil and Gas Producers $\left.{ }^{[3]}\right)$.

While it is rare to find properly documented data from which incident rates can be developed, there have been some attempts at petrochemical incident rate data collection stretching several decades. These, of course, come out of regulatory systems that mandate detailed incident reporting, as well as data collection about the equipment in service within a given system. Examples of such systems include the federally-regulated pipeline industry in the United States ${ }^{[4]}$ and the offshore industry in the United Kingdom (UK).

The UK system, founded after the Piper Alpha incident, mandates incident reporting for all hydrocarbon releases from offshore systems, as well as recording of the equipment populations of all installations. This hydrocarbons release database (HCRD) is maintained by the Health and Safety Executive (HSE), and has collected data since $1992^{[5]}$, continuing under the Reporting of Injuries, Diseases and Dangerous Occurrences Regulations (RIDDOR) since 2013. It has become the chosen database for petrochemical QRA applications due to its comprehensive nature, where all hydrocarbon releases, and the populations of equipment from which releases could happen (and have happened), are captured by the data gathering requirements.

\section{Risk Criteria}

There are, effectively, two ways to use QRA results. The first is in a comparative way. In a comparative QRA, two or more options are evaluated and the outcome of the analysis provides the basis for the risk assessment in the end. In this situation, the various results (associated with the various options) are compared to each other to see which option creates a lower risk. This approach does not typically concern itself with the absolute value of calculated risk, just the risk relative to the other options. In taking this viewpoint, the uncertainties of the analysis can be ignored (as they are deemed equal) and the insufficiencies of either the frequency data or the consequence analysis may be overlooked - provided that the same methodology and assumptions were applied to each option.

In contrast to comparative QRA, most studies are done so that their results can be compared to some criterion in the risk assessment stage (after the analysis is complete) to determine if the calculated risk is acceptable or not. In this case, a criterion or criteria are needed to evaluate the risk. It is this application for which many governmental or industry (and some corporate) criteria have been developed. These criteria allow risk analysts to compare calculated risk to a standard to evaluate whether or not the level of risk is acceptable. 
When developing measures of risk, there needs to be some baseline time frame on which the risk is calculated. For comparative risk, this is perhaps open to the analyst, as the only item of importance in the end is the comparison itself. With published risk criteria, the basis is virtually always one (calendar) year. In this way, the calculated risk has a time constraint and can be compared to other measures with the same time constraint. In most cases, published risk criteria are based on fatalities for members of the public. Thus, the risk criteria sets an acceptable limit for the public's exposure to a potentially fatal event within a one year period. This common basis also allows for comparison to other modes of potential fatality, which are often expressed on an annual basis.

\section{Frequency vs. Probability}

Within the context of conducting QRAs, there is often debate on the exact definitions (and meaning of) frequency and probability. While mathematicians may have very precise definitions for these terms, they become blurred when put into the context of a petrochemical QRA. So, for the purposes of this paper, the following definitions will apply ${ }^{[6]}$ :

Frequency: The number of occurrences of an event per unit of time.

Probability: The likelihood of an event or event sequence during an interval of time; expressed as a number ranging from 0 to 1 .

Using these concepts, it is possible to establish the basis for a QRA in both terminology and the numerical formulation of frequencies and probabilities. The QRA then proceeds with the following steps:

- Historical data is gathered for the events of concern. In this case, each incident is some type of loss of containment (LOC) that results in a hydrocarbon (or other chemical) release.

- Using the number of incidents over a given number of years, as well as the population count for the equipment that could have had an LOC, an incident rate is developed. This incident rate is expressed as the number of incidents per year, for a given equipment type.

- Incident rates are applied as LOC event frequencies to evaluate specific hazardous events.

- Frequencies may be modified by conditional probabilities, which are a value between zero and one, addressing the occurrence of some specific condition, provided that an initiating even has occurred. Examples include the probability of given hole size when there is a release event, the probability of certain weather conditions among the many site-specific possibilities, or the probability of ignition of the flammable vapor cloud that is generated.

- The LOC frequency (as modified by several probabilities) is mathematically transformed into the probability of the unique event in a one-year period for use in the risk calculations. While probabilities do not by definition carry units of time, it is necessary to address the probability values with a specific time period (i.e., one year) due to the basis of the incident rates, as well as the basis for risk assessment through comparison to established criteria.

With the above process outlined, it can be seen that it is necessary to use historical frequencies, expressed as incident rates, to define probabilities that are then used in QRA calculations. The remainder of this paper focuses on the development of frequencies associated with petrochemical processing equipment incidents that represent a LOC event. 
The first use of a database is to develop a total incident rate for each type of equipment. This rate is independent of the specifics of each incident (such as release magnitude or release hole size) as it is only concerned with the number of incidents experienced by a given type of equipment. The total incident rate (TIR) can be easily expressed as:

$$
T I R=\frac{\text { number of incidents }}{\text { Population,equipment } \cdot \text { years }}
$$

Consider the following example. In a given installation, there are 200 incidents involving LOC events from pumps during a ten-year span that data is gathered. If the population of pumps is 1,000 for every year over the same span of 10 years, then there are 10,000 pump-years in the data set. Application of Equation 1 then provides the total incident rate:

$$
T I R(\text { pumps })=\frac{200 \text { incidents }}{10,000 \text { pump-years }}=2.0 \times 10^{-2} \text { incidents per year, per pump }
$$

In this example, pumps would be found to have a historical incident rate of 0.02 per year, or $2.0 \times 10^{-2}$ per year. This incident rate can then be used for probability calculations in a QRA. The calculated frequency, or incident rate, can be transformed into a probability using the following formula:

$$
P=1-e^{(-\lambda \cdot t)}
$$

Where:

$$
\begin{aligned}
& P=\text { annual probability of occurrence (dimensionless) } \\
& \lambda=\text { annual incident frequency (LOC events per year) } \\
& t=\text { time period (one year) }
\end{aligned}
$$

This transformation provides the probability that a given event will occur within a specified time period. When dealing with incident rates for QRA and subsequent comparison to published standards, the time period is always set at one year. Because of this, the incident rates must be expressed as LOC events per year. While probabilities are dimensionless, we attach the word 'annual' to the probability to signify that it is the probability of the event happening in the period of one year.

For our example above, the incident rate for pumps, per year, was found to be $2.0 \times 10^{-2}$. This results in an annual probability of $1.98013 \times 10^{-2}$. This means that if the historical data are taken to be applicable to future pump events, in any given year there is a one in 50.5 chance of any individual pump experiencing an LOC event. Note that, numerically, the incident rate is nearly identical to the probability. This is true for frequencies less than about $1.0 \times 10^{-2}$, but diverges as the frequency approaches one. For events with a frequency of once per year, the annual probability is about 0.632. For the typical frequencies (incident rates) used in a QRA, the frequency and probability values are often close enough such that they are effectively interchangeable. 


\section{HCRD}

As discussed above, the most comprehensive database for LOC events is the HSE's HCRD. Each incident recorded in the database is a release of hydrocarbon, without regard to the cause of that event. Additionally, there is a full count of the equipment from which LOC events could occur, broken down by equipment types. The reporting for both events and population is mandatory, overseen by regulators in the UK. Reporting began in 1992 and has continued consistently since then. As of the writing of this paper, the data through 2016 was the latest available ${ }^{[7]}$.

The HCRD is effectively two databases: (1) a recording of equipment populations, and (2) a reporting $\log$ for incidents. The equipment population data is reported annually, and includes classification by one of 53 systems and by equipment in one of 120 categories or subcategories. Incidents include any unintended release of hydrocarbons, and each incident is reported with a set of information including the time, date, place, and other circumstances associated with the release event, the material released, the amount released, pressure at the time of the release, the hole size, the cause of the release, and many other parameters. The database includes a total of 4,756 incident entries for 1995 through 2016.

There have been previous attempts to provide LOC event data. In 2013, Det Norske Veritas (DNV) published a guidelines document that provided frequency data for use in QRAs ${ }^{[8]}$. The data, extracted from the HCRD, was presented for a range of equipment sizes within the various equipment types, for a fixed set of hole size ranges. Then recently, Lloyd's Register published a revision for the Process leak for offshore installations frequency assessment model $\operatorname{PLOFAM}(2)^{[9]}$, which is based on the offshore Norwegian Continental Shelf facilities that are similar to the UK's offshore facilities. Both of these publications present LOC event data, but the former is somewhat cumbersome, and the latter is a complicated system whose results are geared more toward mass release rates than incident rates. Thus, this paper strives to provide a more streamlined and flexible system for event probability determination.

\section{Calculating Equipment Incident Rates}

The HCRD provides a recording of all hydrocarbon release incidents from the population of equipment within its scope of influence. Each release event is reported under the HSE's system, and data is gathered concerning the release. Of the many data fields in the database, this analysis was concerned with the following entries for each recorded incident:

- HCRD ID (a unique identification number)

- The HSE-ranked incident severity (minor, major, significant)

- Equipment codes (general, primary, secondary, and tertiary classifications)

- Estimated quantity released [kg]

- Equivalent hole diameter [mm]

- Pressure at the time of release [bar]

Of the 4,756 recorded release events in the database, there were several that did not have an entry (often "BLANK" is found), or had a bad entry, in the equipment code field(s). Without a valid 
equipment code, these incidents were not able to be categorized, and were excluded from the analysis.

The core consideration in this analysis was that each incident be of sufficient magnitude such that the event is capable of harming people or damaging equipment. Many of the incidents are reported to have occurred at very low pressures or to have released very little hydrocarbon. So, while such events have been reported as LOCs, they are not considered to be a hazardous incident for the purposes of incident rates applied to a QRA. These are often referred to as "maintenance events" where certain maintenance tasks result in hydrocarbon release even after the system has been depressurized or partially drained/vented. The following criteria were applied to the data:

- Remove incidents where a valid equipment code is not provided (326 incidents removed)

- Remove incidents whose reported release pressure was less than 0.5 bar, unless that incident was labeled by the HSE as major or significant (267 incidents removed)

- Remove incidents whose reported released mass was less than $1.0 \mathrm{~kg}$, unless that incident was labeled by the HSE as major or significant (461 incidents removed)

The labeling of 728 incidents as maintenance events represents the removal of about $15 \%$ of the incidents from consideration. While the pressure and released mass filtering values are somewhat arbitrary, they are deemed appropriate due to the concerns listed above. Figure 1 demonstrates the independent distributions of mass released and release pressure within the HCRD.

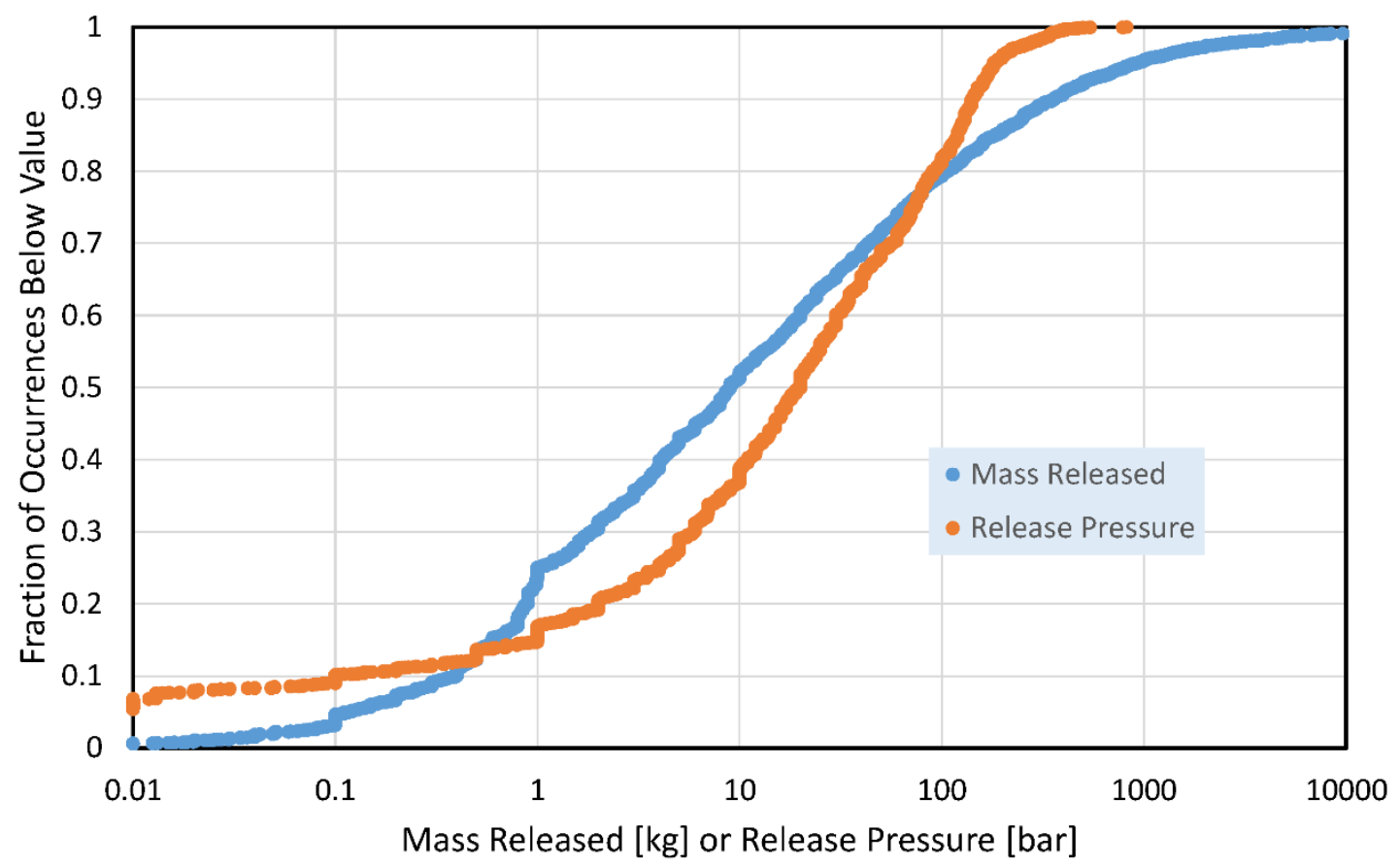

Figure 1

Cumulative Distribution of Mass Released and Release Pressure Data

Of the 120 categories and sub-categories of equipment types, there are about 30 classifications or equipment groups that are of general interest to QRAs. In many cases, multiple equipment types 
are grouped to develop a more general incident rate. For example, the HCRD includes 38 classifications for valves, based on various classifications by valve size (diameter), whether they are manual or actuated, or whether the valve is a blowdown, choke, emergency shut down, relief, or bleed valve. From these 38 categories, a user could determine an incident rate for a very specific valve type (if there were sufficient incidents with that type; sometimes there are none), but it is more statistically meaningful to group valves into larger categories. In this work, valves are divided into classifications of manual or actuated. All valve characteristics or service types are lumped into these two categories.

The results for the total incident rate calculation are given in Table 1, based on a selection of equipment types that is generally useful to QRA application for hydrocarbon processing systems.

Table 1

Calculated Equipment Incident Rates

\begin{tabular}{||l|c|c|c||}
\hline Equipment Type or Group & $\begin{array}{c}\text { HCRD Number } \\
\text { of Equipment- } \\
\text { Years }\end{array}$ & $\begin{array}{c}\text { Number of } \\
\text { Incidents } \\
\text { (Filtered) }\end{array}$ & $\begin{array}{c}\text { Calculated Total } \\
\text { LOC Rate per } \\
\text { Year }\end{array}$ \\
\hline \hline Compressors & 8,661 & 106 & $1.224 \times 10^{-2}$ \\
\hline Crude Oil Storage & $8,733.5$ & 59 & $6.756 \times 10^{-3}$ \\
\hline Filters & $19,435.6$ & 59 & $3.036 \times 10^{-3}$ \\
\hline Flanged Connections & $13,747,657$ & 379 & $2.575 \times 10^{-5}$ \\
\hline Flexible Connections & $1,787,286.3 \dagger$ & 135 & $7.553 \times 10^{-5} \dagger$ \\
\hline $\begin{array}{l}\text { Shell \& Tube Heat } \\
\text { Exchangers }\end{array}$ & $20,630.2$ & 47 & $2.278 \times 10^{-3}$ \\
\hline Plate Heat Exchangers & 6,690 & 47 & $7.025 \times 10^{-3}$ \\
\hline All Heat Exchangers & $29,329.1$ & 102 & $3.478 \times 10^{-3}$ \\
\hline $\begin{array}{l}\text { Instruments (Small } \\
\text { Connections) }\end{array}$ & $1,629,769$ & 641 & $3.933 \times 10^{-4}$ \\
\hline Pig Traps & 8,160 & 36 & $4.412 \times 10^{-3}$ \\
\hline Piping D $\leq 3$ inches & $3,600,856 \dagger$ & 512 & $1.422 \times 10^{-4} \dagger$ \\
\hline Piping 3 < D $<11$ inches & $6,093,607 \dagger$ & 245 & $4.021 \times 10^{-5} \dagger$ \\
\hline Piping D $\geq 11$ inches & $1,918,542 \dagger$ & 69 & $3.596 \times 10^{-5} \dagger$ \\
\hline Pressure Vessels & $44,715.6$ & 82 & $1.834 \times 10^{-3}$ \\
\hline Pumps & $28,246.7$ & 176 & $6.231 \times 10^{-3}$ \\
\hline Turbines & $3,222.4$ & 121 & $3.755 \times 10^{-2}$ \\
\hline Actuated Valves & 724,192 & 375 & $5.178 \times 10^{-4}$ \\
\hline Manual Valves & $3,744,845$ & 277 & $7.397 \times 10^{-5}$ \\
\hline All Valves & $4,469,037$ & 644 & $1.441 \times 10^{-4}$ \\
\hline \hline
\end{tabular}

$\dagger$ Reported count by meters of piping; incident rate per meter per year 
While the HCRD offers data on many equipment types, there are some that are not included and must be referenced in outside databases. Incident frequency data for items such as large floating roof storage tanks, LPG transfer hoses, LNG marine transfer hard arms, and others do not appear in the HCRD. The methodologies presented in this paper could be applied to these other items, but they are not discussed further here.

\section{Hole Size Distribution}

While the development of total incident rate is important, it does not fully describe the frequency of an LOC event. When applied to a properly constructed QRA, incident rate data must include a relationship between event hole size and the total incident rate. This is generally realized as large, high consequence events having small frequencies (or probabilities) and small, low consequence events having larger frequencies, such that within the total incident rate, there is a probabilistic distribution of hole sizes that create the range of consequences.

To develop a hole size distribution from the HCRD, the analysis began with the set of incidents used for the total incident rate. A second filtering was applied on the reported hole size so that a numerical evaluation of hole sizes could be done. All incident entries that had no hole size identified (listed as "BLANK") or a hole size of "999" mm were removed from the analysis. This filtering reduces the data set, but only in the sense that incidents with undefined hole sizes are not used to describe the hole size distribution. The "999" values are a database placeholder where a real hole size was not recorded, and thus are not discarding holes of nearly one meter in diameter.

If the data remaining in the analysis are grouped by equipment type, then any one group can be evaluated on the range of hole sizes that were reported in the HCRD. At this level of analysis, no other variables are important to the analysis - only the individual hole sizes and how any one relates to the distribution of hole sizes within the full set are of interest. Distilling the data to this level leaves a list of hole sizes and a total number of incidents for that equipment type. The most useful way to characterize this data set is a cumulative distribution, which describes the fraction of total incidents as a function of hole size. Such a relationship can then be applied to the various hole sizes modeled in a QRA to calculate the probability of a given hole size range, which is then represented by a single hole size and matched with the appropriate consequence modeling set. Analysis of the data found that a two-variable log-logistic equation was able to sufficiently describe the fraction of incidents as a function of hole size. The log-logistic cumulative distribution function $(\mathrm{CDF})$ can be expressed as:

$$
C=\frac{1}{1+\left(\frac{d}{\alpha}\right)^{-\beta}}
$$

Where:

$\mathrm{C}=$ Fraction of occurrences for hole sizes up to hole size $d$

$\mathrm{d}=$ hole diameter, $\mathrm{mm}$

$\alpha=$ alpha parameter for log-logistic equation

$\beta=$ beta parameter for log-logistic equation 
In this application, for a given hole size, $d$, the CDF represents the fraction of all LOC events that will have a hole size of that diameter or smaller. When combined with the total incident rate (as calculated above) the CDF can be used to describe the frequency of LOC events in a given hole size range:

$$
f_{d_{2}-d_{1}}=T I R \cdot\left(C_{2}-C_{1}\right)
$$

Where:

$$
\begin{aligned}
& \mathrm{C}_{1}=\text { Fraction of occurrences for hole sizes up to hole size } \mathrm{d}_{1} \\
& \mathrm{C}_{2}=\text { Fraction of occurrences for hole sizes up to hole size } \mathrm{d}_{2} \\
& \mathrm{TIR}=\text { Total incident rate for the equipment type } \\
& f_{d_{2}-d_{1}}=\text { LOC event frequency for hole size range between } \mathrm{d}_{1} \text { and } \mathrm{d}_{2}
\end{aligned}
$$

To implement Equation 5, the analyst must first have the alpha and beta parameters that best fit the data for a given type of processing equipment. An example plot for pump LOC events from the HCRD is shown in Figure 2, with the hole size axis show in log-scale. The graph in Figure 2 demonstrates the hole size distribution for multiple pump types, as well as the distribution of all pump incidents as a single data set, and how a log-logistic curve fits this data.

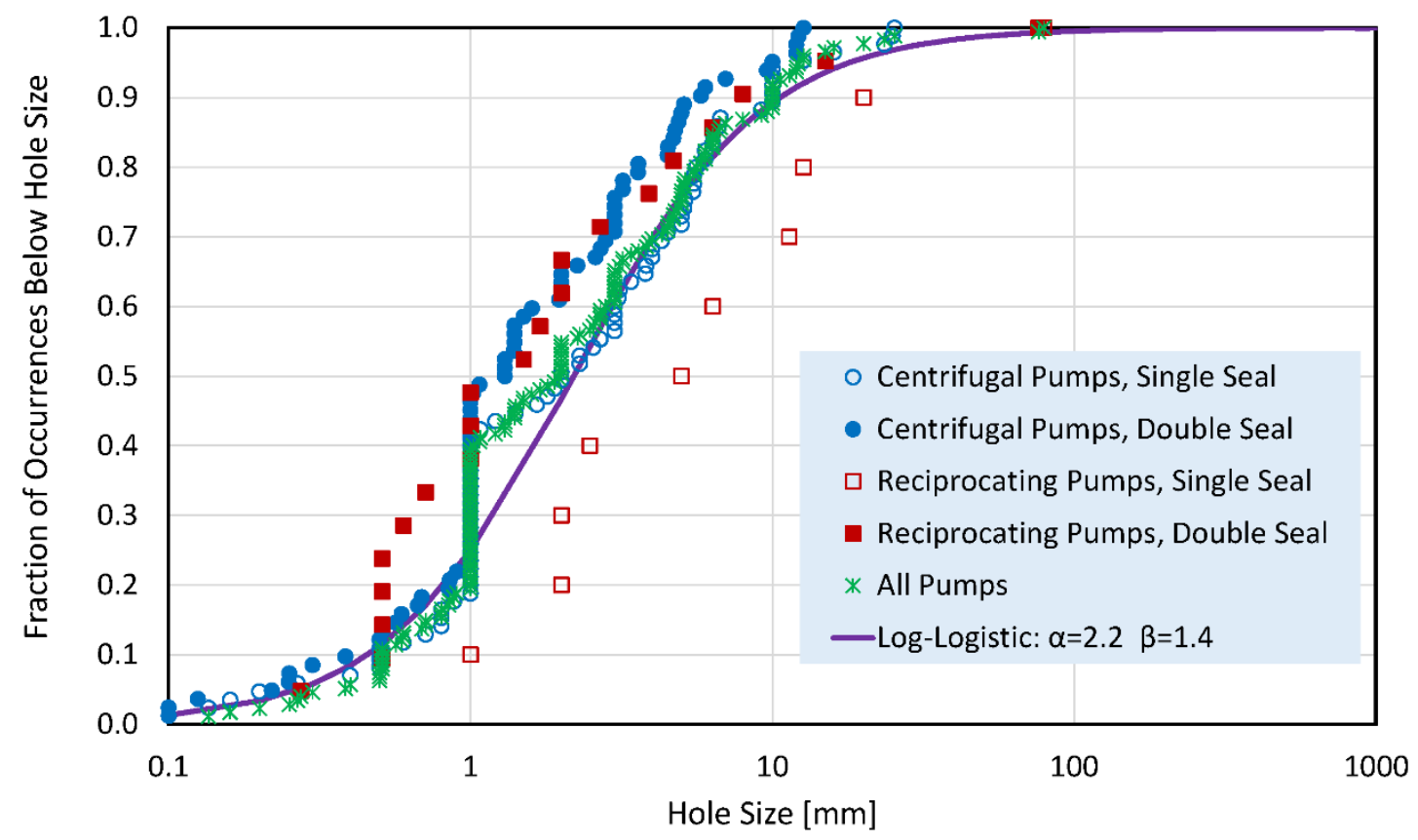

Figure 2

\section{Cumulative Distribution of Hole Size and Log-Logistic Curve Fit for Pumps}

A review of Figure 2 shows that the four types of pumps for which data is collected in the HCRD each have a unique distribution. This data could be used to create a log-logistic fit for each pump classification in the database. The problem that occurs is that some data sets are not large enough to be reliably used. For example, as seen in Figure 2, the single seal reciprocating pumps only have ten recorded incidents, and are not statistically significant for their own curve fit. It is better to merge these pumps with the double seal reciprocating pumps (21 incidents) or create a group 
that is all pumps (175 incidents), as shown in Figure 2. While there is a discernable difference between each of the four pump types, and between single or double seals, a combined group is a reasonable representation of all pump types.

The implementation of a log-logistic curve fit has specific implications when the results are interpreted. As an example, consider the log-logistic fit in Figure 2, where 25\% of the occurrences are predicted to be $1 \mathrm{~mm}$ or smaller holes, $50 \%$ are predicted to be $2 \mathrm{~mm}$ or smaller, and about $90 \%$ are predicted to be $10 \mathrm{~mm}$ or smaller. This indicates that $90 \%$ of the total incident rate is attributed to the smaller hole sizes. The fit to one curve for multiple equipment classifications also shows the potential imprecision of the method. Considering the single seal reciprocating pumps, the $50 \%$ value for is about $5 \mathrm{~mm}$, and the $90 \%$ value at $20 \mathrm{~mm}$. If there were sufficient data to develop an independent curve fit for single seal reciprocating pumps, and that expanded data followed the same trend indicated in Figure 2, it may be warranted to have a separate curve.

\section{Application to QRA}

The system described here is implemented by having a table of values, by equipment type, listing the total incident rate and the alpha and beta parameters for the hole size distribution. To select incident rates for the various hole sizes that apply to a type of equipment, a risk analyst can follow these steps:

- Define a set of hole sizes to be implemented in the QRA where

- Each chosen hole size defines a ranges of incident hole sizes;

- For the smallest hole size range, the $d_{1}$ value is taken to be zero; and

$\circ$ The largest range is categorized as a rupture and ends with a very large hole (e.g., $1 \mathrm{~m})$

- Select the equipment type.

- For each hole size range, use Equations 4 and 5 above to assign an LOC frequency for the hole size range, using the total incident rate for that type of equipment, the alpha and beta parameters, and the bounding hole sizes.

- Where there is a maximum hole size for a type of equipment (e.g., instrument connections) the hole size range representing the maximum hole size should be assigned a frequency that is the sum of the calculated one for that range and all larger hole sizes. All larger hole size ranges being applied in the QRA should then be assigned a frequency of zero.

- Select the consequence modeling hole size associated with the specified hole size range (e.g., a range of 15-25 $\mathrm{mm}$ can be modeled with a $25 \mathrm{~mm}$ diameter hole). The hole size at the top of a range is often applied as a conservative assumption, but a hole size in the middle of the range may also be selected.

A set of values that can be used with the above methodology is given in Table 2, including the alpha and beta parameters for the various equipment types.

\section{Concluding Remarks}

The system presented in this paper represents a portable and flexible tool that can be applied to various QRA studies. It offers the comprehensive basis of the HCRD, plus a simplified 
methodology for application with a QRA. Table 2 can be applied to various QRA studies where the hole sizes selected for analysis are varied. This offers consistency in the applied incident rate data, as well as consistency in the hole size distribution.

This methodology can be followed with a different set of assumptions applied to HCRD, or alternatively, the methodology can be applied to other databases that have the same quality of data. The latter option would certainly be necessary for equipment types not found in the HCRD.

Table 2

Incident Rates and Cumulative Distribution Factors

\begin{tabular}{||l|c|c|c||}
\hline \multirow{2}{*}{ Equipment Type or Group } & $\begin{array}{c}\text { Calculated } \\
\text { Total Incident } \\
\text { Rate per Year }\end{array}$ & \multicolumn{2}{c|}{$\begin{array}{c}\text { Log-Logistic } \\
\text { Curve Fit } \\
\text { Parameters }\end{array}$} \\
\cline { 3 - 4 } & & $\boldsymbol{\alpha}$ & $\boldsymbol{\beta}$ \\
\hline \hline Compressors & $1.224 \times 10^{-2}$ & 2 & 1.3 \\
\hline Crude Oil Storage & $6.756 \times 10^{-3}$ & 18 & 1.2 \\
\hline Filters & $3.036 \times 10^{-3}$ & 16 & 1.1 \\
\hline Flanged Connections & $2.575 \times 10^{-5}$ & 1.5 & 0.9 \\
\hline Flexible Connections & $7.553 \times 10^{-5} \dagger$ & 5.5 & 1.2 \\
\hline Shell \& Tube Heat Exchangers & $2.278 \times 10^{-3}$ & 1.3 & 1 \\
\hline Plate Heat Exchangers & $7.025 \times 10^{-3}$ & 3 & 1.7 \\
\hline All Heat Exchangers & $3.478 \times 10^{-3}$ & 2 & 1.3 \\
\hline Instruments (Small & $3.933 \times 10^{-4}$ & 3.5 & 1.9 \\
\hline Connections) & $4.412 \times 10^{-3}$ & 2 & 0.9 \\
\hline Pig Traps & $1.422 \times 10^{-4} \dagger$ & 2 & 1.2 \\
\hline Piping D $\leq 3$ inches & $4.021 \times 10^{-5} \dagger$ & 1.8 & 0.8 \\
\hline Piping 3 < D <11 inches & $3.596 \times 10^{-5} \dagger$ & 2 & 0.7 \\
\hline Piping D $\geq 11$ inches & $1.834 \times 10^{-3}$ & 8 & 1 \\
\hline Pressure Vessels & $6.231 \times 10^{-3}$ & 2.2 & 1.4 \\
\hline Pumps & $3.755 \times 10^{-2}$ & 2 & 1.2 \\
\hline Turbines & $5.178 \times 10^{-4}$ & 1.3 & 0.9 \\
\hline Actuated Valves & $7.397 \times 10^{-5}$ & 2 & 0.9 \\
\hline Manual Valves & $1.441 \times 10^{-4}$ & 1.7 & 0.9 \\
\hline All Valves & & \\
\hline
\end{tabular}

$\dagger$ Incident rate per meter per year 


\section{References}

[1] Marx, J.D. and J.B. Cornwell (2008), "The Importance of Weather Variations in a Quantitative Risk Analysis." Presented at Mary Kay O'Connor Process Safety Center Annual Symposium, College Station, Texas, October 28-29, 2008.

[2] TNO (2005), Guidelines for Quantitative Risk Assessment, the Purple Book. CPR 18E, the Netherlands Organization for Applied Scientific Research, Committee for the Prevention of Disasters, the Hague, Netherlands, December 2005.

[3] OGP (2010), Occupational Risk Assessment Data Directory, Report No. 434-1. Process release frequencies. The International Association of Oil \& Gas Producers. March 2010.

[4] PHMSA (2019), Distribution, Transmission \& Gathering, LNG, and Liquid Accident and Incident Data. Pipeline and Hazardous Materials Safety Administration, Washington, DC, Updated March 7, 2019.

https://www.phmsa.dot.gov/data-and-statistics/pipeline/distribution-transmissiongathering-lng-and-liquid-accident-and-incident-data (accessed September 2019)

[5] HSE (2019), Hydrocarbon Releases System. Health and Safety Executive, United Kingdom.

http://www.hse.gov.uk/offshore/hydrocarbon.htm (accessed September 2019)

[6] CCPS (2000), Guidelines for Chemical Process Quantitative Risk Analysis - Second Edition. Center for Chemical Process Safety of the American Institute of Chemical Engineers, 3 Park Avenue, New York, New York, 10016. (ISBN 0-8169-0720-X)

[7] HSE (2019), Statistics. Health and Safety Executive, United Kingdom. http://www.hse.gov.uk/offshore/statistics.htm (accessed September 2019)

[8] DNV (2013), Failure Frequency Guidance - Process Equipment Leak Frequency Data for Use in QRA. Det Norske Veritas AS, Norway, www.dnv.com.

[9] PLOFAM (2018), Process Leak for Offshore Installations Frequency Assessment ModelPLOFAM(2), Lloyds' Register Group Limited, Report No. 107566/R1, December 6, 2018. (https://www.norskoljeoggass.no/contentassets/9972d6e9f1b74f158931aac3b462770e/10 7566_r1_final.pdf) 